were to determine etiological agents of UD syndrome among males attending STI clinics and associated risk factors.

Methods We conducted a cross-sectional study among males presenting with complaints of dysuria and/or urethral discharge at eight government and non-governmental organization STI clinics in four Indian states from 2008 to 2009. A behavioural questionnaire was administered, clinical examination performed and urine was collected to test for Neisseria gonorrhoeae (GC) and Chlamydia trachomatis (CT) by Gen-Probe-APTIMA Combo II. In cases where a urethral discharge was elicited, urethral swabs were collected and tested for Mycoplasma genitalium (MG), Ureaplasma urealyticum (UU) and Trichomonas vaginalis (TV) by using PCR method. Data were analysed using STATA V10.

Results 246 clients diagnosed with UD syndrome as per the national algorithm were enrolled in the study. $29 \%$ of the participants had at least one of the infections. The overall prevalence of GC was 14\% and CT was $4 \%$. Among the 86 participants from whom urethral swabs were collected, prevalence of MG and UU was 33\% and 34\% respectively while TV was not detected. In this sub-group of 86 participants, the prevalence of GC and CT was higher at $24 \%$ and $7 \%$ respectively while $64 \%$ individuals had at least one infection. Factors such as younger age ( $<25$ years), illiteracy, paid sex in last 2 weeks and penetrative anal sex in last 3 months were found to be significantly associated with having any infection (see Abstract P3-S1.20 table 1).

Abstract P3-S1.20 Table 1 Correlates of infections (gonococcal and/or non-gonococcal)

\begin{tabular}{llll}
\hline S. No. & Characteristic & OR $(\mathbf{9 5 \%}$ Cl) & p Value \\
\hline 1 & Age up to 25 years & $1.7(0.9$ to 3.1$)$ & 0.06 \\
2 & Inability to read or write & $2.2(0.9$ to 5.1$)$ & 0.05 \\
3 & Paid sex in last 2 weeks & $2.1(1.1$ to 4.2$)$ & 0.02 \\
4 & Penetrative anal sex in past 3 months & $2.1(0.9$ to 4.8$)$ & 0.05 \\
5 & Vaginal sex in past 3 months & $2.8(0.6$ to 26.4$)$ & 0.16 \\
6 & No condom use at last sexual act & $1.4(0.7$ to 2.7$)$ & 0.32 \\
\hline
\end{tabular}

Conclusion The current practice of diagnosing UD syndrome based on the history of dysuria and/or discharge is leading to over-diagnosis. A detailed sexual history for determining risk factors and demonstration of urethral discharge on clinical examination will help to improve the diagnosis.

\section{P3-S1.21 NON-CULTURE BASED NEISSERIA GONORRHOEAE ANTIMICROBIAL RESISTANCE SURVEILLANCE}

\section{doi:10.1136/sextrans-2011-050108.421}

${ }^{1} \mathrm{~N}$ Goire, ${ }^{2} \mathrm{~K}$ Freeman, ${ }^{3} \mathrm{~J}$ Tapsall, ${ }^{1} \mathrm{~S}$ Lambert, ${ }^{4} \mathrm{M}$ Nissen, ${ }^{1} \mathrm{~T}$ Sloots, ${ }^{1} \mathrm{D}$ Whiley. ${ }^{1}$ Queensland Children's Medical Research Institute/Sir Albert Sakzewski Virus Research Centre, Brisbane, Australia; ${ }^{2}$ Royal Darwin Hospital, Darwin, Australia; ${ }^{3}$ Prince of Wales Hospital, Sydney, Australia; ${ }^{4}$ Pathology Queensland Central, Royal Brisbane and Women's Hospital Campus, Brisbane, Australia

Background Increased reliance on nucleic acid amplification tests for the diagnosis of gonorrhoea, and issues with transporting viable Neisseria gonorrhoeae (NG) isolates, particularly from remote regions, undermines bacterial-culture-based NG antimicrobial resistance (AMR) surveillance. In this study, we explored non-culture based NG AMR surveillance by developing and validating a real-time PCR assay for direct detection of penicillinase-producing NG (PPNG) in clinical samples.

Methods The PPNG-PCR assay was designed as an indirect marker of penicillinase activity, by targeting a region of sequence conserved across all NG plasmid types harbouring the $\beta$-lactamse gene, while not targeting the actual $\beta$-lactamase encoding sequence. The assay was evaluated using 118 characterised NG clinical isolates, and then applied to samples collected from the Australia's Northern Territory (years 2008-2009) where penicillin is still used for treatment. These comprised 214 NG-positive clinical samples from which $N$ gonorrhoeae were isolated and phenotypic penicillinase results were available and an additional 209 samples that were positive by NG-PCR only.

Results The PPNG-PCR2 assay provided 100\% sensitivity and $98.5 \%$ specificity compared to bacterial culture results for the detection of PPNG in clinical specimens. PPNG-PCR false-positive results, presumably due to cross reaction with unrelated bacterial species, were observed in four clinical samples but were distinguished on the basis of late cycle threshold values. A total of 15/423 (3.6\%) samples were positive by PPNG-PCR. These data vary from phenotypic surveillance rates for this region $(2.5 \%-2.9 \%)$.

Conclusion In tandem with phenotypic surveillance, the PPNG-PCR assay provides enhanced epidemiological surveillance of $N$ gonorrhoeae penicillin resistance and is of particular relevance to regions where penicillin is still used to treat gonorrhoea. We are currently evaluating assays targeting NG chromosomally-mediated resistance mechanism to $\beta$-lactam antibiotics.

\section{P3-S1.22 EVALUATION OF PERFORMANCE OF SIX COMMERCIAL ASSAYS FOR DETECTION OF CHARACTERISED ISOLATES OF NEISSERIA GONORRHOEAE AND OTHER NEISSERIA $S P P$.}

doi:10.1136/sextrans-2011-050108.422

${ }^{1} \mathrm{~S}$ Tabrizi, ${ }^{2} \mathrm{M}$ Unemo, ${ }^{3} \mathrm{~A}$ Limnios, ${ }^{4} \mathrm{~S} 0$ Hjelmevoll, ${ }^{1} \mathrm{~S}$ Garland, ${ }^{3} \mathrm{~J}$ Tapsall. ${ }^{1}$ The Royal Women's Hospital, Parkville, Australia; ${ }^{2}$ Örebro University Hospital, Örebro, Sweden; ${ }^{3}$ The Prince of Wales Hospital, Randwick, Australia; ${ }^{4}$ University Hospital of North Norway, Tromsö, Norway

Background Molecular detection of Neisseria gonorrhoeae in urogenital samples is now routinely conducted in many diagnostic laboratories with the demand also expanding to testing of extragenital samples. Testing of extragenital samples poses a challenge as it may result in false positive results due to cross-reaction with commensal Neisseria spp. and $N$ meningitidis. Adequate evaluation of molecular assays is essential prior to expanding utilisation of the assays to such specimens.

Method This study aimed to examine 450 characterised clinical isolates comprising of $216 \mathrm{~N}$ gonorrhoeae and 234 other Neisseria spp. and closely related bacteria from various geographical regions worldwide with six commercial assays including GenProbe APTIMA Combo 2 and APTIMA GC; Roche COBAS Amplicor CT/NG and COBAS 4800 CT/NG; BD ProbeTec GC Ox Amplified DNA Assay and Abbott RealTime CT/NG.

Results Among $216 \mathrm{~N}$ gonorrhoeae isolates included in the study, all assays except COBAS Amplicor where four (1.9\%) gonococcus isolates were not detected, showed a positive result with all $N$ gonorrhoeae isolates. Among 234 Neisseria spp. evaluated, initial results showed all assays evaluated to display cross reaction with non-gonococcal Neisseria isolates. COBAS Amplicor and ProbeTec showed highest number of false positives, detecting $33(14.1 \%)$ and 26 (11\%) non-gonococcal Neisseria isolates respectively. Abbott RealTime, APTIMA Combo and APTIMA GC, and Roche COBAS 4800 showed initially low level of cross reaction, that is, detected 2 $(1 \%), 5(2.1 \%), 4(1.7 \%)$ and $2(1 \%)$ of the isolates respectively. When retested by the company using a fresh culture, none of these nine isolates showed cross reaction with the respective assays.

Conclusion COBAS Amplicor and ProbeTec displayed highest number of false positives among assays evaluated, with the remaining assays only showing sporadic low level false positivity. Especially when examining extragenital specimens, supplementary testing for all assays and platforms remains recommended. 